\title{
Current Status and Progress of the Treatment of Limited-Disease Small Cell Lung Cancer
}

\author{
Minghui Xu, Jiaxiang Wang*, Zhigang Zhou, Lu Ling, Mingyue Yang \\ School of Medicine, Shandong University, Jinan 250012, China \\ *Corresponding author: Jiaxiang Wang, 211253058@qq.com
}

\begin{abstract}
Small cell lung cancer (SCLC) is a common pathological type of primary lung cancer originating from the bronchial mucosa or gland. SCLC is characterized by rapid growth, high degree of malignancy, and early metastasis, which poses a great threat to patients' safety and quality of life. SCLC can be divided into two stages: localized disease and extensive disease. For limited-disease small cell lung cancer (LD-SCLC), radiotherapy and chemotherapy are often used in clinical treatment. In recent years, there are several new advances in the clinical treatment of SCLC, including the improvement of radiotherapy and chemotherapy methods, compatibility of first-line and second-line drugs, as well as immune-targeted therapy. This article discusses the current status of clinical treatment and the research progress of LD-SCLC in the past five years.
\end{abstract}

Keywords: Small-cell lung cancer; Bureau within a time limit; Chemotherapy; Radiation therapy; Immunotherapy

Publication date: September 2021; Online publication: September 30, 2021

\section{Introduction}

Small cell lung cancer (SCLC) is a common pathological type of primary lung cancer originating from the bronchial mucosa or glands. Its prevalence accounts for about $15 \%$ of lung cancer ${ }^{[1]}$. Compared to nonsmall cell lung cancer (NSCLC), SCLC is characterized by rapid disease progression, short natural course, high degree of malignancy, early metastasis and recurrence, poor prognosis, as well as short survival period, of which the five-year survival rate of patients is only about 5\% ${ }^{[2]}$. Therefore, the research and clinical application of the treatment of SCLC is of great significance to control patients' condition and improve their quality of life.

SCLC is divided into two clinical stages: localized disease and extensive disease. In SCLC, the tumor is usually confined to one thoracic cavity in addition to supraclavicular or scalenus anterior lymph node involvement and ipsilateral pleural effusion. For limited-disease small cell lung cancer (LD-SCLC), a further step in clinical staging should be done based on TNM staging in order to improve the accuracy for individualized treatment. SCLC is characterized by small lesions, easy metastasis, and sensitive to radiotherapy and chemotherapy. In regard to that, radiotherapy and chemotherapy are more commonly used as treatment compared to surgery. In recent years, there several new advances in the clinical treatment of SCLC, including radiotherapy and chemotherapy methods, first-line and second-line treatment regimens, as well as immunotherapies and targeted therapies. In this article, the common clinical treatment methods and the research progress of LD-SCLC in the past five years would be discussed along with the prospects of the treatment of LD-SCLC in the future. 


\section{Definition and traditional treatment of LD-SCLC}

\subsection{Definition of LD-SCLC}

The current clinical therapy for SCLC according to the standard consensus of staging by the International Association for the Study of Lung Cancer (IASLC) can be expressed as the bureau within a time limit. In LD-SCLC, the tumor is confined to one side of the chest cavity and the drainage area of mediastinal lymph nodes, as well as bilateral and supraclavicular lymph nodes. The involvement of ipsilateral pleural effusion, recurrent laryngeal nerve invasion, and superior vena cava obstruction are also part of the limited disease. There are hardly any clinical comparisons or discussions regarding the earlier and more severe staging, the current IASLC staging, and the commonly used TNM staging for patients undergoing surgical treatment for this disease. However, it is generally believed that the time limit of localized SCLC can also be considered the same as stage I of TNM for clinical surgical treatment.

\subsection{Traditional treatment methods for LD-SCLC}

Only patients with TNM stage IA, clinically and stage IB, pathologically can receive surgical treatment. Therefore, less than 5\% of patients with localized small cell lung cancer can receive surgical treatment. For the rest of the patients, if there is no lymph node metastasis, single chemotherapy can be used. Patients with lymph node metastasis should receive concurrent radiotherapy and chemotherapy. For patients with LDSCLC, the traditional first-line chemotherapy regimen is cisplatin plus etoposide. For patients who are intolerant to cisplatin, carboplatin may be used instead as alternative therapy. During the first or second cycle of chemotherapy, radiotherapy can be concurrently carried out. If radiotherapy is performed twice a day, the dose is 1.5 Gy per time and the cumulative dose is $45 \mathrm{~Gy}$. If radiotherapy is performed once a day, the dose is 1.8-2.0 Gy per time, and the cumulative dose is 60-70 Gy. In regard to the traditional treatment of SCLC, although this method has a certain therapeutic effect and it can effectively control the development of the disease, it is easy for patients to develop tolerance, follow-up recurrence risk, and considerable side effects. This is exemplified with the use of etoposide; as one of the first-line treatment of SCLC, its toxicity can aggravate lung injury. In addition, there have been cases of treatment-related acute myeloid leukemia caused by chemoradiotherapy ${ }^{[3]}$.

\section{Current status and progress of the treatment of LD-SCLC in the last five years}

\subsection{Current status of the treatment}

In addition to surgical treatment and postoperative chemotherapy for a small number of patients who truly meet the TNM staging of lung cancer at stage IA and IB, etoposide plus cisplatin (or) carboplatin combined with thoracic radiation therapy are commonly used in clinical practice for most patients with SCLC. For patients with complete or partial remission, prophylactic brain irradiation is often followed to reduce the risk of brain metastases. Studies of thoracic radiotherapy regimens have shown that early concurrent hyperfractionated radiotherapy combined with chemotherapy can improve the prognosis of patients with LD-SCLC ${ }^{[4]}$. Although the first-line treatment for patients provides certain relief, there are disadvantages. First-line chemotherapy drugs may cause several adverse reactions in patients, especially for those with carboplatin and cisplatin intolerance; they could be serious or even life-threatening for frail patients. In addition, a considerable number of patients would relapse within 1 to 2 years after receiving the first-line treatment due to the development of drug resistance. Second-line treatment can then be used at this time, but the effect of second-line treatment is often poor. At present, it is difficult to eradicate the cancer and prolong the survival time with non-surgical treatment of LD-SCLC.

However, the development of medicine does not stop here. In recent years, some immune-targeted drugs and biological agents have opened a new path for the treatment and as adjuvant therapies for SCLC, 
but some of them are still in clinical trials and have not been widely used as first-line treatment.

\subsection{Progress of the treatment}

\subsubsection{Lurbinectedin - a new drug for the treatment of SCLC}

Lurbinectedin is an alkylating agent that binds to guanine residues in DNA to trigger a series of reactions that lead to the apoptosis of cancer cells ${ }^{[5]}$. In addition, it can induce RNA polymerase II degradation and inhibit RNA synthesis during transcription ${ }^{[6]}$. It can also selectively reduce the number of macrophages and blood vessels in the tumor tissue and change the tumor microenvironment, which have a positive effect in anti-cancer ${ }^{[7]}$.

During its phase I clinical trial, fatigue, nausea, vomiting, loss of appetite, mucositis, hair loss, diarrhea, constipation, and other non-hematological adverse events were found in the subjects. There were also adverse hematological events such as neutropenia, leukopenia, anemia, thrombocytopenia, etc. It may result in mild to moderate abnormalities in patients' biochemical markers, such as increased creatinine, alanine aminotransferase, and aspartate aminotransferase. Moreover, the drug can also cause bone marrow suppression and liver damage along with a $34 \%$ incidence of serious adverse events, including pneumonia, respiratory infections, anemia, dyspnea, and a reduction in various blood components. Therefore, further studies and the verification of the efficacy and safety of lurbinectedin are still required.

\subsubsection{Irinotecan combined with magnetic induction hyperthermia - a new combination therapy}

Magnetic induction hyperthermia $(\mathrm{MIH})$ is a new technology that irradiates magnetic medium into the tumor site by alternating magnetic field to produce heat and destroy the tumor tissue ${ }^{[8]}$. In recent years, because the side effects of magnetic induction hyperthermia on patients are far less than those of drug therapy and radiotherapy, this medical technology has gradually become a new research hotspot and a new method for tumor diagnosis and treatment under the aforementioned combined strategy in the field of clinical anticancer ${ }^{[9,10]}$.

Tumor tissues are less heat-resistant than normal tissues in the body; when the temperature reaches $43^{\circ} \mathrm{C}$ $-45^{\circ} \mathrm{C}$, tumor cells can be damaged or even undergo apoptosis. In view of that, magnetic induction hyperthermia, as a breakthrough, came into being ${ }^{[11]}$. In recent years, magnetic induction hyperthermia has been proven to have the effect of chemotherapeutic sensitization. Professor Li Lia's team, from the Department of Oncology of Integrated Traditional Chinese and Western Medicine of China-Japan Friendship Hospital, conducted a clinical trial to investigate the effect of irinotecan combined with magnetic induction hyperthermia on SCLC H446 cells. This experiment confirmed that magnetic induction hyperthermia can inhibit the proliferation and promote the apoptosis of $\mathrm{H} 446$ cells with an additive antitumor effect when combined with irinotecan ${ }^{[12]}$.

\subsubsection{Immunotherapy combined with other forms of treatment}

In recent years, Li Meng's team, from Jinzhou Medical University, studied the effect of CHIP (C-terminus of heat shock protein 70 interacting protein) overexpression on human small cell lung cancer cell lines. In this experiment, small cell lung cancer cell lines were cultured in vitro, and the relationship between CHIP and MET expression in these cell lines was determined by western blotting. This experiment concluded that CHIP inhibited MET-dependent signaling, regulated MET-mediated SCLC movement, apoptosis, and invasion, as well as significantly reduce cell invasion and growth while promoting cell apoptosis through its overexpression in SCLC cell lines ${ }^{[13]}$. This method has not step into the clinical trial phase. There are differences between in vitro culture and in vivo environment, so it has not been popularized. However, it is a new way to fight cancer. 


\subsubsection{Other anticancer strategies}

In 2019, Lu Jiarong's team found that serum markers such as pro-gastrin-releasing peptide (proGRP) provide evidence for the treatment of SCLC, which may offer new ideas for anticancer ${ }^{[14]}$.

\section{Future prospects for the treatment of LD-SCLC}

At present, controlling LD-SCLC in patients more effectively, delaying the development of the disease, improving the five-year survival rate of patients with SCLC, reducing the side effects of various treatments as much as possible, and improving patients' quality of life are difficult problems in the prevention and treatment of thoracic tumors. The existing clinical treatment scheme and current research have made considerable progress. However, several existing research results have not been promoted and some have not even completed clinical trials.

In view of this, it is necessary to further promote the research progress of various clinical trials and explore new paths for treatment. These are undoubtedly of great significance for the treatment of LD-SCLC.

\section{Disclosure statement}

The authors declare that there is no conflict of interest.

\section{References}

[1] Carbone DP, Morgensztern D, Le Moulec S, et al., 2018, Efficacy and Safety of Rovalpituzumab Tesirine in Patients with DLL3-Expressing, $\geqslant 3^{\text {rd }}$ Line Small Cell Lung Cancer: Results from the Phase 2 TRINITY Study. J Clin Endocrinol, 36(15): 8507.

[2] Wu LY, 2016, Study on the Antitumor Effect of PLK1 Inhibitor on Small Cell Lung Cancer and Reversal of Chemotherapy Resistance. J Clin Cancer, 2016, 19(06): 409-412.

[3] Ji Q, Liu Z, Li H, et al., 2021, Acute Myeloid Leukemia Induced by Chemotherapy and Radiotherapy in Localized Small Cell Lung Cancer. Journal of Applied Cardiovascular, Cerebral and Pulmonary Diseases, 29(01): 118-121.

[4] Zhou X, Li L, 2007, Progress in the Treatment of Small Cell Lung Cancer. Cancer Progress, (05): 475-488.

[5] Shinn LT, PharmD, Vo KA, et al., 2020, Lurbinectedin: A New Treatment Option for Relapsed/Refractory Small-Cell Lung Cancer. Annals of Pharmacotherapy, 55(9): 1172-1179.

[6] Santamaria Nunez G, Robles CMG, Giraudon C, et al., 2016, Lurbinectedin Specifically Triggers the Degradation of Phosphorylated RNA Polymerase II and the Formation of DNA Breaks in Cancer Cells. Mol Cancer Ther, 15(10): 2399-2412.

[7] Belgiovine C, Bello E, Liguori M, et al., 2017, Lurbinectedin Reduces Tumor-Associated Macrophages and the Inflammatory Tumor Microenvironment in Preclinical Models. Brit J Cancer, 117(5): 628-638.

[8] Fan L, Zhong X, Tang J, et al., 2021, Effect of Irinotecan Combined with Magnetic Induction Hyperthermia on Cell Proliferation, Apoptosis and Cycle of Small Cell Lung Cancer. Modern Oncology Medicine, 29(09): 1471-1475.

[9] Chen L, Wu Y, Wu H, Et al., 2019, Magnetic Targeting Combined with Active Targeting of DualLigand Iron Oxide Nanoprobes to Promote the Penetration Depth in Tumors with Effective Magnetic Resonance Imaging and Hyperthermia. Acta Biomaterialia, 96: 491-504.

[10] Yu X, Ding S, Yang R, 2021, Research Progress on Magnetic Nanoparticles for Magnetic Induction Hyperthermia of Malignant Tumor. Ceramics International, 47(5): 5909-5917. 
[11] Bhardwaj A, Parekh K, Jain N, 2020, Invitro Hyperthermic Effect on Cervical and Breast Cancer Cells. Sci Rep, 10.

[12] Phung DC, Nguyen HT, Phuong Tran TT, 2019, Combined hyperthermia and chemotherapy as a synergistic anticancer treatment. Journal of Pharmaceutical Investigation, 49: 519-526.

[13] Li M, Zhang J, Li D, Effects of Heat Shock Protein 70 C-Terminal Reactive Protein on Human Small Cell Lung Cancer Cell Line. Journal of Jinzhou Medical University, 201, 42(01): 25-29, 34.

[14] Lu JR, Application of Gastrin-Releasing Peptide Precursor in the Diagnosis and Treatment of Small Cell Lung Cancer. Journal of Mathematical Medicine and Pharmacy, 201, 34(03): 328-330. 